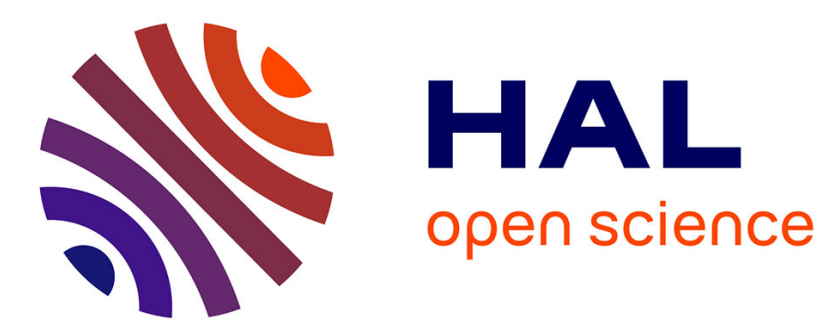

\title{
Manger du Chien à Tahiti: une Affirmation Identitaire?
}

Christophe Serra-Mallol

\section{To cite this version:}

Christophe Serra-Mallol. Manger du Chien à Tahiti: une Affirmation Identitaire?. Anthropozoologica, 2010, 45 (1), pp.157-172. 10.5252/az2010n1a11 . hal-02970507

\section{HAL Id: hal-02970507 https://hal.science/hal-02970507}

Submitted on 18 Oct 2020

HAL is a multi-disciplinary open access archive for the deposit and dissemination of scientific research documents, whether they are published or not. The documents may come from teaching and research institutions in France or abroad, or from public or private research centers.
L'archive ouverte pluridisciplinaire HAL, est destinée au dépôt et à la diffusion de documents scientifiques de niveau recherche, publiés ou non, émanant des établissements d'enseignement et de recherche français ou étrangers, des laboratoires publics ou privés. 
Pré-print of Serra-Mallol Christophe, 2010. "Manger du chien à Tahiti : une affirmation identitaire ?", Anthropozoologica , 45-1, p. 157-172.

DOI : $10.5252 /$ az2010n1a11

\title{
Manger du chien à Tahiti : une affirmation identitaire ? Eating dog in Tahiti : an identary affirmation ?
}

\author{
SERRA-MALLOL Christophe
}

Résumé : La rareté des animaux terrestres, l'importance symbolique conférée à la viande rouge, et la stratification sociale marquée par des interdits alimentaires nombreux, ont fait du chien un aliment de choix dans l'ensemble de l'aire polynésienne pré-européenne. Aliment sacrificiel destiné en offrande aux dieux et à la consommation cérémonielle, support de rites d'échanges et de conciliation, réservé à l'élite de la société ancienne, sa consommation a été relevée à Tahiti par les premiers Européens en contact avant qu'elle ne soit désacralisée par les missionnaires anglais et français. Malgré les nombreux changements qui ont marqué l'évolution de la Polynésie française au cours des deux derniers siècles, et le discrédit porté sur le chien comme aliment, la consommation de chien est occasionnelle aujourd'hui mais toujours présente parmi la population locale. Une enquête sur les pratiques et représentations alimentaires menée en zone urbaine à Tahiti et Moorea a permis de mettre en évidence la permanence de la consommation périodique de viande de chien de la part de groupes sociaux spécifiques. La préparation d'un plat à base de chien fait l'objet d'un choix raisonné de l'animal destiné à être abattu, d'un apprêt préalable et d'un mode de cuisson particuliers, et d'un mode de préparation culinaire original. Réprouvée par la population d'origine métropolitaine et par les autorités publiques, la viande de chien constitue un des marqueurs gustatifs ethniques au fondement d'une identité ma'ohi en pleine reconstruction.

Mots clés: anthropologie de l'alimentation, habitudes alimentaires, cynophagie, Polynésie, Tahiti, chien, identité

Abstract: The scarcity of the ground animals, the symbolic importance conferred on the red meat, and the social stratification marked by numerous food prohibitions, made of the dog a food of choice in the whole of the preEuropean Polynesian area. Sacrificial food intended in offering for the gods and for ceremonial consumption, support of rites of exchanges and conciliation, reserved for the elite of the ancient society, its consumption was raised in Tahiti by the first Europeans in contact before being deconsecrated by the English and French missionaries. In spite of the numerous changes which marked the evolution of French Polynesia during the last two centuries, and the depreciation of $\operatorname{dog}$ as food, the consumption of dog is occasional today but always presents among the local population. A survey on food habits and representations led in urban zone to Tahiti and Moorea allowed to bring to light the durability of the periodic consumption of dog meat on behalf of specific social groups. The preparation of a dish for base of dog makes the object of reasoned choice by the animal intended to be shoots down, of a preliminary apprêt and a particular mode of cooking, and of an original mode of culinary preparation. Disapproved by the population of metropolitan origin and by the public authorities, dog meat constitutes one of the ethnic gustative markers in the foundation of ma'ohi identity in reconstruction.

Key words : anthropology of food, food habits, cynophagy, Polynesia, Tahiti, dog, identity

Les pratiques et représentations alimentaires des individus s'inscrivent dans un système alimentaire dans lequel s'insère le mangeur, système fondateur de l'unité de la société ou du groupe social où chaque individu trouve sa place (Fischler 1990 : 69). Les pratiques alimentaires d'une société donnée ne sont donc pas seulement des lieux d'affirmation de l'identité individuelle, mais s'inscrivent au cœur même du processus sans cesse renouvelé de construction 
de l'identité sociale. Image de soi et d'autrui qui permet de se situer dans les rapports avec les autres, de se classer et de classer autrui dans des groupes dans lesquels s'effectuent ces rapports, l'identité sociale se constitue à partir des objets consommés, et notamment alimentaires, grâce à leur dimension symbolique (Lahlou 1998). L'identité peut être ainsi saisie non pas comme une simple transmission de normes et de valeurs, mais comme un ensemble de représentations que l'individu se construit à partir de sources multiples pour donner du sens à ses pratiques et à la vie (Lahire 2001), l'identité étant précisément ce qui structure cette unité de sens à un moment donné (Kaufmann 2004).

$\mathrm{Au}$ sein du vaste espace insulaire du Pacifique, l'aire polynésienne est composée de sociétés issues de migrations successives de l'ouest vers l'est à partir d'une aire géographique commune située dans le sud-est du continent asiatique. Les sociétés polynésiennes anciennes sont vues comme des sociétés de type «végétaliste » (Barrau 1991 : I 1294-1295 ; Pollock 1992 : 2 ; Saura 2003 : 125), marquées par une vision religieuse et «ethno-écosystémique » de la plante et de la « terre-mère », dans lesquelles la place de la viande animale, accessoire au quotidien, est centrale dans les occasions cérémonielles selon des rites et des normes de préparation et de partage très précis, notamment autour du porc, du chien et de la tortue.

A Tahiti, le contact régulier avec les Européens à partir du dernier quart du dix-huitième siècle, la christianisation généralisée de la société au cours du siècle suivant, la colonisation française et son influence sur l'organisation socio-économique des modes de production, et la structuration d'un commerce de proximité au cours du dernier siècle, ont favorisé l'accès à des biens alimentaires nouveaux ou auparavant interdits. Le système alimentaire tahitien s'en est trouvé modifié, et les pratiques alimentaires également, se métissant en une «tradition » qui mêle aspects anciens et nouveaux. Cette «tradition» alimentaire a été sans cesse remodelée et réinventée jusqu'au mangeur tahitien contemporain ${ }^{1}$. La consommation de viande de chien fait partie de cette «tradition », dans un processus de construction d'une identité culturelle fortement revendiquée aujourd'hui, sans cesse battue en brèche par des visiteurs européens pour lesquels cette viande ne faisait pas partie de leur propre domaine du mangeable.

\section{1- Le chien dans la consommation alimentaire des anciens Polynésiens}

\section{1-1 La place du chien parmi les autres animaux terrestres}

Avant l'arrivée des premiers Polynésiens, il n'existait pas de mammifères terrestres sur les îles isolées du Pacifique oriental. Les seuls animaux terrestres ont tous été apportés par les premiers Polynésiens au cours de leurs migrations transocéaniques de l'ouest vers l'est du Pacifique (Oliver 2002 : 80-82). Ils sont au nombre de trois : le porc, le chien, et la volaille, en sus du rat, et encore tous n'ont pas survécu ou ne sont pas adaptés sur les différentes îles polynésiennes.

En sus du porc réservé aux occasions cérémonielles, les chiens et les poules composaient la majeure partie des animaux terrestres consommés à Tahiti, et parfois les seuls dans certaines îles polynésiennes, à l'exception de l'île de Pâques et de Tongatapu où les chiens étaient absents (Kirch 1991 : 123). L'absence de diversité en matière de chair d'animal terrestre, et notamment de grands herbivores (Farb et Armelagos 1985: 193; Harris 1987:182) a sans doute été un facteur qui a induit les Polynésiens à manger du chien ; le chien et le porc étant par ailleurs

\footnotetext{
${ }^{1}$ Nous entendons par tahitien le groupe social résidant dans les Iles de la Société, et se définissant et fonctionnant suivant des valeurs dites tahitiennes ou plus largement ma'ohi (Saura, 2004 : 119-137), groupe auprès duquel nous avons conduit la recherche dont est tiré le présent article
} 
culturellement comparables (Milliet 1995 : 79). Le nom donné au chien est similaire dans toute la Polynésie : en Nouvelle-Zélande il se nomme kuri, à Samoa uli, à Fidji koli, et à Tahiti 'uri.

Le chien apparaît dans les mythes anciens de toute la Polynésie, Rey-Lescure (1946: 269) signale ainsi l'existence à Tahiti d'un dieu chien nommé Taahiti, et du dieu Irawanu en NouvelleZélande, «père du chien ». A Tahiti, il était censé protéger les hommes par son aboiement (Henry 2000 : 394). Il faisait partie des offrandes des cérémonies hautement sacrées, ou touchant à la survie du groupe, comme lors des cérémonies qui suivaient la mort du chef ou ari'i, ou encore dans le rite de conciliation 'utu pendant lequel les deux partis échangeaient deux jeunes chiens lorsque la paix était conclue entre belligérants.

Les chiens présents à Tahiti à l'arrivée des Européens sont décrits par Forster (1777 : 378 cité par Oliver 1974: I 276) comme petits avec une tête large, le museau pointu, de petits yeux, des oreilles dressées, et un poil plutôt long de couleur blanche et marron. Il les trouve timides et peu agressifs, ce que certains expliquent par leur nourriture, à base de végétaux -fruits de l'arbre à pain, noix de coco, ignames... (Rey-Lescure 1946: 268 ; Harris 1987 : 181)- plutôt que de viande. Chez les anciens Tahitiens, les restes de préparation non consommables (arêtes de poissons, viscères...) étaient jetés dans une fosse faisant office de poubelle, et recouverte de terre ensuite, ou destinés à la nourriture des cochons et des chiens s'il s'agissait de restes végétaux (pelures de taro et de 'uru, cœur de 'uru...).

Par manque de gibier, les chiens n'étaient pas utilisés pour la chasse en Polynésie, à l'exception de la Nouvelle-Zélande (Oliver 2002 : 82) où les relations entre humains et chiens devaient être d'un autre ordre du fait de leur rôle dans l'approvisionnement alimentaire (Ellen 1999).

Les porcs, chiens et poules étaient laissés en semi liberté à Tahiti et dans les Iles de la Société, les deux premiers pouvant être nourris par leurs propriétaires. On ne trouve que peu de descriptions d'élevage de porcs ou de chiens dans les sources primaires, l'élevage de porcs comme des chiens étant pourtant un élément commun aux cultures polynésiennes ancestrales (Oliver 1974 : II 853), et se retrouve dans tout le Pacifique sud sauf en Nouvelle-Calédonie, porcs et chiens étant élevés à la fois pour la nourriture et le prestige tiré de leur possession et de leur circulation. Des gardiennages de porcs et de chiens sont ainsi avérés parmi les chefs ou ari'i à proximité des marae, temples où ils étaient destinés à être sacrifiés (Ferdon 1981 : 195). Les chiens appartenant aux ari'i et aux prêtres leur étaient vraisemblablement réservés, marqués comme les porcs d'un interdit ou tapu signifié matériellement par une ficelle dans l'oreille.

Dans les cas où les porcs étaient rares ou inexistants comme à Hawaii, les chiens jouaient le rôle du porc ailleurs (Kirch 1991 : 123 ; Milliet 1995 : 79), et étaient également nourris des produits de l'agriculture, bruts ou même préparés comme le poi $^{2}$ pour les engraisser (Titcomb 1969 : 6-8 ; Handy et Handy 1972 : 245). Plutôt élevés en liberté autour des habitations aux Iles de la Société, ils l'étaient à Hawaii sur des terrains appropriés où des abris leur étaient construits pour qu'ils puissent y dormir (Oliver 2002 : 234-235).

Comme les porcelets, ils pouvaient être utilisés comme animaux de compagnie par les anciens Polynésiens (Barrau 1983: 125), jusqu'à parfois allaiter les chiots au sein à Tahiti (Forster 1777 : 378 cité dans Oliver 1974 : I 276 ; Wilson 1968 : 361), comme on le signale également à propos de Hawaii (Titcomb 1969 ; Handy et Handy 1972 : 243), au grand dégoût des Européens.

\footnotetext{
${ }^{2}$ Le poi ou popoi est une forme de préparation semi-liquide ou plus compacte sous forme de crème du fruit de l'arbre à pain, combinaison du fruit de l'arbre à pain frais et du mahi (fruit de l'arbre à pain de la saison passée préparé en pâte fermentée) battue sur une petite table basse avec une pierre réservée à cet usage, et mélangée avec de l'eau. A ce mélange dosé en fonction du goût individuel et de la disponibilité de fruits frais peuvent être ajoutés de la banane ou autres fruits pour sucrer le mélange.
} 
Ils pouvaient dans ce cas jouer le rôle de «tétaïre », tétant les femmes dont la production de lait était trop abondante voire douloureuse (Pujol et Carbone 1991 : 1347-1348).

\section{1-2 Un aliment réservé}

La viande de chien était considérée comme supérieure à celle des porcs (Molyneux 1988 : 558 ; Parkinson 1982 : 20 ; Wilson $1968: 360-361$ ), et pouvait constituer la principale viande lors des repas de cérémonie (Ferdon 1981 : 196 ; Harris 1987 : 181), ainsi que le mode de «taxation » le plus utilisé pour les prestations faites aux chefs (Milliet 1995: 86; Oliver 2002:234-235) et notamment à Hawaii. Du fait de leur relative rareté et de leur valeur d'échange importante, les mammifères terrestres ne faisaient pas l'objet d'une consommation quotidienne : il s'agit d'aliments « de luxe ${ }^{3}$ (Leach 2003 : 447), réservés à une élite et/ou à des occasions rituelles de consommation. Porcs et chiens, ainsi que les volailles même si de façon plus secondaire, étaient à Tahiti comme dans l'ensemble de la Polynésie d'abord destinés aux dons rituels et aux festins qui suivaient les cérémonies religieuses (Pollock 1992 : 2).

L'affirmation générale de Philippe Raust (1986 : 100) du chien comme apport carné principal ${ }^{4}$ en Polynésie n'est pas vérifiée dans la littérature (Harris 1987 : 181), sauf pour Hawaii et à propos de la seule affirmation de Ferdon (1981 : 89) quant à la possibilité d'en manger par les gens du commun également. Des travaux archéologiques ont d'ailleurs montré que les os de cochons sont plus fréquents que ceux de chiens dans les Iles de la Société (Titcomb 1969 : 30).

La présence violente du sacrifice dans la société tahitienne place la consommation de viande, de porc ou de chien, sur le plan du festif et du cérémoniel et l'éloigne de l'alimentation quotidienne, dans laquelle n'intervient pas le rapport scénique, essentiel lors du sacrifice (Pujol et Carbone 1991: I 1362). Potentiellement émanation d'un esprit (Henry 2000: 394), le chien était essentiellement un élément cérémonial et sacrificiel comme l'indique la description ${ }^{5}$ par Anderson de la cérémonie sur un marae qui fait suite à la mort d'un chef. Bougainville (1982 : 494) et Cook (1998 : 60) les signalent en tant qu'offrandes aux dieux sur un marae à Tahiti, ainsi que Dumont d'Urville (1988 : 494) aux Marquises.

Les aliments destinés à la consommation rituelle des dieux étaient interdits aux femmes : viande rouge dont le porc, le chien et la tortue, les poissons du large, et certaines variétés du fruit de l'arbre à pain 'uru, de noix de coco et de bananes (Bougainville 1982 : 252 ; Anderson in Scemla 1999 : 826 ; Wilson 1968 : 145). Ces interdits portaient essentiellement sur les aliments d'origine

\footnotetext{
${ }^{3}$ Certains produits alimentaires utilisés par les anciens Polynésiens peuvent être définis comme «produits de luxe » s'ils répondent à un des critères suivants : s'ils sont réservés à une élite, servis dans des ustensiles décorés, importés de l'extérieur, disponibles rarement, préparés à l'aide d'une combinaison complexe d'ingrédients. De plus, pour figurer convenablement dans un festin, ces produits doivent être assemblés en quantités très importantes, être dotés d'une plus-value avérée en matière de préparation ou de préservation, être reconnus comme une variété supérieure, et/ou être servis en même temps dans des modes de préparation variés (Leach 2003 : 454). La notion « d'aliment de luxe » peut donc parfaitement s'appliquer au chien, comme au porc et à la tortue.

${ }^{4}$ Dans l'Encyclopédie de la Polynésie française Philippe Raust écrit « Le chien constituait l'apport carné essentiel, à l'exception du poisson, bien avant le porc pour la population de base» (1986: II 100).

${ }^{5}$ « During this time a Dog has been brought and was killed by twisting his neck and suffocating him, after which a fire being made on the spot the hair was singed off and the entrails taken out. The guts were burnt on the fire but the liver only roasted, and the body of the dog after being besmeared with blood and dried over the fire was with the liver put on a whatta or scaffold about six feet high, along with two dogs and two hogs which had lately been sacrified and at this time emitted an intolerable stench. While this operation was performing the hiests repeated a mournful prayer and now and then put some more bits of red fathers on one of the bundles of cloth; a boy who assisted at the same time called out several times in a very shrill doleful tone, which we were informed was to invite their god to come and feed on the sacrifice, and when the dog was put upon the whatta the priests and attendants gave a kind of shout which closed the ceremony » (Anderson 1988 : III 979-980).
} 
animale, comme dans d'autres parties du monde ${ }^{6}$. Toutefois, il semble que les porcs élevés, préparés et cuits par les femmes de la classe des chefs et leur appartenant pouvaient être consommés par ces dernières. Il semble en avoir été de même pour la viande de chien (Oliver 1974 : I 224).

Semblables aux aliments destinés aux dieux, les aliments réservés aux chefs étaient essentiellement les aliments les plus appréciés et les plus recherchés : 'uru bien sûr, taro, mais surtout les animaux à viande rouge, comme le porc, le chien, et également la tortue et les grands poissons pélagiques du large sous forme de présent sacré $i$ 'a ra'a roroa (Oliver 1974 : II 1005). Les dons de ces aliments étaient fortement codifiés dans leur forme et dans le statut de leurs destinataires : chefs, familles de chefs, artisans spécialistes.... Porcs et chiens vivants faisaient partie des dons ou du tribut faits aux chefs; après la cérémonie d'offrande, ils étaient rendus à leurs propriétaires pour qu'ils les engraissent en les laissant à la libre disposition des chefs.

Le chef ari'i étant sacré, tous les aliments, végétaux et surtout animaux, frappés d'interdits lui étaient réservés de droit. Il pouvait les faire partager à l'occasion de certaines cérémonies en faisant circuler le sacré avec les autres participants à partir de festins à base de tortue, de porc ou de chien. Consommer de la chair animale et notamment de la viande rouge était ainsi réservé au sacré. Comme l'indique Colette Méchin (1997: 121-123) à propos de l'Europe de l'Ouest, la viande, de porc ou de gibier, mais aussi de chien et de tortue à Tahiti, n'est jamais « neutre », sans signification : elle est un privilège, un attribut de classe, en Polynésie comme ailleurs, et de tout temps.

Lors de la préparation du chien comme celle du porc, l'animal était soigneusement tué, par strangulation ou étouffement, pour préserver intact son corps, avant d'être enduit de son sang et lui donner ainsi la couleur sacrée des plumes d'oiseaux utilisées pendant la cérémonie, puis cuit dans un four du marae, lieu de culte où avait lieu la cérémonie, sur un feu appelé ahi ha'apena (feu sacré) avant d'inviter les dieux au repas. A l'occasion des sacrifices, le dieu honoré pouvait exprimer sa part d'animalité : manger le festin qui lui était offert, le restant partagé ensuite entre les « invités » et les personnes présentes à la cérémonie.

Le chien était également utilisé comme viande cérémonielle aux îles Samoa et à Hawaii, de façon encore plus importante qu'à Tahiti : Ellis (1972 : II 869) indique que la chair du chien constituait la viande principale à l'occasion de ces festins, et a pu compter jusqu'à quatre cents chiens rôtis avec autant de porcs et de poissons au cours d'un seul festin.

Si les chiens étaient surtout utilisés pour leur chair, leurs dents ornaient parfois les vêtements de cérémonies, leurs os servaient à fabriquer des hameçons de pêche (Ferdon 1981: 195; Oliver 2002 : 82), et leur fourrure à confectionner des capes pour les chefs maori (Harris 1987 : 181-

182). Les poils de chien étaient utilisés pour élaborer des espèces de perruques, les plus longs poils servant de franges au taumi, ornement pectoral des guerriers ma'ohi (Rey-Lescure 1946 : 270).

\section{1-3 La perception de la consommation tahitienne de chien par les premiers Européens}

Le four polynésien enterré ou ahima'a était d'usage pour nourrir un nombre important de commensaux, ou pour la cuisson d'un animal de grande taille, comme les porcs ou les chiens. Cook nous donne dans un passage devenu fameux la recette du chien au four polynésien à l'occasion d'un don fait par une cheffesse de Tahiti :

\footnotetext{
${ }^{6}$ Sur 800 interdits recensés en Afrique, 740 portent sur la viande selon Igor de Garine (1991 : I 1507).
} 
«Il fallait accepter les présents qu'elle nous avait apportés, à savoir : un cochon, un chien, quelques fruits à pain, et des bananes. Nous avions refusé le chien, en donnant la raison que nous n'avions pas besoin de ce genre d'animaux; cela parut l'étonner un peu, et elle nous dit que sa chair était très bonne à manger ; nous eûmes sans tarder l'occasion de constater que cela était vrai, car, Monsieur Banks ayant acheté un panier de fruits parmi lesquels se trouvait une cuisse de chien apprêtée pour être mangée, plusieurs d'entre nous y goûtèrent et trouvèrent que cette viande était très estimable. Ils prirent donc le chien d'Obiriha et le firent séance tenante apprêter par quelques naturels de la façon suivante : ils firent d'abord dans la terre un trou profond d'un pied environ, et y allumèrent un feu dans lequel ils firent chauffer quelques petites pierres. Pendant ce temps on étrangla le chien et on lui arracha les poils en le plaçant à plusieurs reprises sur le feu, et il arriva à être aussi propre que si on l'avait échaudé à l'eau bouillante, on le vida et les entrailles furent parfaitement lavées avec tout le reste, et aussitôt que le trou et les pierres furent assez chauds, on éteignit le feu et on laissa une partie des pierres au fond du trou. Sur ces pierres on posa des feuilles vertes, et sur ces feuilles le chien, les boyaux par-dessus, et le tout fut recouvert de feuilles ; après quoi le trou fut parfaitement refermé avec du terreau. Après être resté ainsi quatre heures, le four -c'est le mot qui convient- fut ouvert et on en sortit le chien, entier et cuit à point. » (Cook 1998 : 48-49).

Après un premier mouvement de refus lié aux habitudes culinaires européennes qui étonne la cheffesse, Cook et ses hommes acceptent par obligation rituelle le don qui leur est fait, après que quelques-uns d'entre eux aient déjà cédé à la consommation de viande canine : ils choisissent de faire cuire immédiatement le chien plutôt que le cochon qui fait également partie du présent. Une fois la cuisson terminée, et ayant goûté à une viande jugée dégoûtante dans leur pays d'origine, les hommes de Cook en apprécient alors la saveur : «Tous ceux qui en avaient goûté déclarèrent qu'ils n'avaient jamais mangé viande plus savoureuse, et que, désormais, ils ne mépriseraient plus la viande de chien » (Cook 1998 : 48-49).

Sydney Parkinson (1984 : 20) rapporte la même anecdote, en modérant toutefois l'enthousiasme culinaire de Cook (Beaglehole 1988 : I 130 note): «I ate a little of it; it had the taste of coarse beef, and a strong disagreeable smell; but Captain Cook, Mr. Banks, and Dr. Solander, commended it highly, saying it was the sweetest meat they had ever tasted; but the rest of our people could not be prevailed on to ate nay of it », et précise que les Tahitiens tirèrent ensuite les boyaux, les mirent sur des pierres chaudes, et dès qu'ils furent grillés, les mangèrent avec avidité. Une fois l'interdit européen franchi, plus par nécessité que par réelle envie, les navigateurs trouvent la viande de chien délicieuse : "Comme animaux domestiques, ils ont des cochons, des poulets et des chiens, auxquels ils nous firent prendre goût, si bien que la plupart d'entre nous placent cette viande au premier rang après les agneaux d'Angleterre » (Cook 1998 : 53), citation que reprend Morrison (1989 : 116) dans son Journal.

L'agréable surprise gustative qu'éprouve Cook en goûtant au chien ne fait pour autant pas disparaître les habitus européens en matière animale, comme en témoigne l'épisode moins connu où malade à bord de son navire, Cook demande qu'on lui fasse cuire le chien favori d'un de ses hommes : «Quand je commençai à me rétablir [de violentes coliques], un chien favori qui appartenait à monsieur (sic) Forster fut offert en sacrifice à mon estomac fragile. Nous n'avions absolument aucune autre viande fraîche à bord, et je pouvais manger de sa chair, et du bouillon qu'on en faisait, alors que je ne pouvais supporter le goût de rien d'autre ; ainsi je puisais de la nourriture et des forces dans un aliment qui aurait soulevé le cœur de la plupart des Européens, tellement il est vrai que nécessité fait loi »(1998: 225). Même s'il s'agit d'un dernier recours, d'une nécessité quasi médicale, Cook n'hésite pas pour autant à «sacrifier » l'animal familier d'un de ses hommes pour le consommer.

En revanche, les espèces à la fois végétales et animales introduites par les premiers navigateurs européens furent plus appréciées par curiosité du fait de leur nouveauté que pour leurs vertus alimentaires, les Tahitiens séparant les couples d'animaux dont beaucoup s'éteignirent ainsi faute 
de reproduction «car chacun voulut avoir sa part des curiosités » (Morrison 1989 : 199). Ainsi « les moutons crevèrent » faute d'être tondus, les bovidés disparurent presque complètement (il en restait un couple à l'arrivée de la Bounty) car les Tahitiens considéraient «ces animaux comme sans valeur au point de vue nourriture », et «avaient une aversion marquée pour les boucs et les chèvres en raison de leur odeur désagréable (...) on les considère comme inférieures à un chien ». Perçue comme répugnante en Europe, la viande de chien était jugée par les Tahitiens comme bien meilleure que celles de nos mammifères caprins européens, rejoignant par là l'avis de Cook

Après les premiers contacts avec les navigateurs, les Tahitiens voient s'installer les missionnaires qui ont pour mission civilisatrice de christianiser les autochtones. Sur les ruines des anciens marae s'élèvent dès 1820 les nouveaux temples, faits de chaux et de blocs de corail. Le nouvel ordre chrétien commence à exercer son influence sur les pratiques organisationnelles et institutionnelles mais également dans la vie quotidienne des Tahitiens. Le caractère sacré de certains aliments est profané par leur consommation publique sous l'injonction des missionnaires, et notamment le rite le plus sacré de l'ancienne religion : la consommation de la tortue, jusque-là réservée aux chefs après une préparation rituelle dans l'enceinte même du temple ancien ou marae. Citées par Dumont d'Urville (1988 : 555-556) et par Ellis, les violations volontaires sont multiples de la part des chefs tahitiens: la préparation de la tortue est faite en dehors de l'enceinte sacrée du marae, sur un feu non dédié, aucun morceau n'est offert en préalable aux dieux en sacrifice, et l'ensemble de l'assemblée invitée à partager ce mets. Le même moyen est utilisé en 1820 à Rurutu aux îles Australes (Ellis 1972 : II 691), et une violation supplémentaire eut lieu, en plus du lieu et du mode de préparation: la séparation sexuelle ne fut plus respectée, puisque femmes et enfants participèrent au repas ${ }^{7}$ et mangèrent des «nourritures interdites », comme le rapporte également Ellis (1972: I 234) à propos de l'île de Tubuai. La même expérience de transgression publique a été relevée à Hawaii avec le roi Kamehameha II (Farb et Armelagos 1985 : 146).

L'objectif des missionnaires était non seulement de désacraliser la consommation de la tortue, nourriture sacrée ou tapu par excellence, mais d'obliger les Tahitiens à renoncer à la consommation de viande de chien comme cela a été le cas aux îles Samoa (Buck 1930 : 127), sans pouvoir pour autant s'appuyer sur les Evangiles muets sur ce point. Comme l'indique Jacqueline Milliet, « de tout temps, c'était faire preuve d'un zèle civilisateur que d'obliger l'Autre à renoncer à ses interdits ". L'absence de cynophagie a souvent ainsi été invoquée comme argument du degré supérieur de civilisation des sociétés occidentales (Milliet 1995: 75-76). Comme l'exprime Colette Méchin (1997: 131) à propos du rôle symbolique des usages alimentaires : « la transgression ostentatoire des usages alimentaires permet (...) de signaler l'abandon pur et simple d'une religion, la rupture avec son groupe d'origine ». La volonté est clairement exprimée et l'entreprise systématique : à travers la transgression du tapu alimentaire le plus strict du système ancien, l'objectif est bien de « détruire le système entier », sa structure, son organisation, et ses modes de relation et de classification.

La désorganisation apparente de la «vie quotidienne », et des «manières » d'être dans ses actes les plus anodins, devient un levier pour soulever vers le dieu chrétien la foi des Polynésiens. Du point de vue des premiers missionnaires on passe d'une société vue comme idéale, paradisiaque, du «bon sauvage », à une vision d'une société déstructurée, anarchique, aux modes de vie

\footnotetext{
${ }^{7}$ A. Babadzan précise que l'un des évangélistes invita les chefs et la population en ces termes : «Chers amis (...) préparez un lieu où nous puissions manger ensemble, vous, vos femmes, vos enfants et votre Roi, en un seul endroit »(Babadzan 1982 : 14-17).
} 
«barbares » et «païens », jusque dans les détails de la vie quotidienne, et l'alimentation en particulier : il s'agissait de faire des Tahitiens, après les Indiens et les Kanaques, des «mangeurs de chiens ». Ellis (1972 : I 317) note à ce propos : «La nourriture, considérée alors comme sacrée, cessa de l'être, les avilissants et odieux interdits attachés aux femmes furent abolis. Et parmi les chrétiens, hommes et femmes purent enfin prendre ensemble de joyeux repas familiaux ».

Le manger a été une des premières cibles des missionnaires protestants britanniques. Le puritanisme et l'ascétisme affichés par les cultures protestantes du nord de l'Europe font que les plaisirs culinaires sont perçus comme grossiers comme le sont les débordements de la chair, et les festins contraires à une certaine idée de la retenue (Garine 1996: 27-28) et plus proches du gaspillage que d'une signification sociale et culturelle. L'ascèse propre au christianisme commence ainsi à être mise en œuvre : jeûne et abstinence périodiques, mise à mort désacralisée et rendue profane des animaux (Flandrin 1996), et notamment du porc auparavant réservé aux occasions rituelles. Les anciens moments centraux de la vie quotidienne sont « digérés » dans les coutumes et jours fériés chrétiens. La société tahitienne se cristallise autour du niveau le plus élémentaire de la famille, la maisonnée, qui donne aux missionnaires l'illusion de recréer un univers parfaitement chrétien.

Peu à peu, les normes occidentales véhiculées au cours des dix-neuvième et vingtième siècles par les classes dirigeantes tahitiennes se sont diffusées en s'adaptant dans la société polynésienne, par une forme de contrôle social basée sur le « savoir-vivre » à l'européenne, qui touchait à la fois les aliments consommés et leur mode de consommation et d'obtention.

\section{2- La consommation de chien aujourd'hui en Polynésie française}

\section{2-1 Une consommation encore avérée}

Après plus de deux siècles de présence européenne, avec la christianisation générale de la société tahitienne au cours de la première moitié du dix-neuvième siècle et la période de colonisation agricole qui l'a suivie, et l'installation du Centre d'Expérimentation nucléaire du Pacifique (C.E.P.) dans les années 1960 avec les transferts financiers massifs qui l'ont accompagné jusqu'à nos jours, qu'en est-il aujourd'hui de la consommation de viande de chien en Polynésie ? Au cours d'une enquête sur les pratiques et représentations alimentaires dans les Iles de la Société ${ }^{8}$, nous avons pu vérifier que la viande de chien est toujours consommée dans un certain nombre de ménages ma'ohi. Sa consommation n'est pas quotidienne mais occasionnelle, notamment pour les fins de semaine, en fonction de la «disponibilité » du «bon » fournisseur, réputé pour savoir choisir et préparer le chien.

L'immuabilité relative de certains plats reflète leur indépendance par rapport au reste du système socioculturel. Ce serait l'autonomie de certains aspects de la cuisine qui leur conférerait une importance particulière, emblématique, aux yeux des individus, notamment quand ils sont placés dans des situations de changement social, et surtout si le changement a été rapide : cela a été le cas du borsch dans l'après-révolution d'octobre en Russie, ou du hamburger dans l'Amérique des années 1950 (Goody 1984 : 248), et du poisson macéré à l'eau de mer et au lait de coco dans le

\footnotetext{
${ }^{8}$ Enquête à base d'entretiens qualitatifs et d'observation participante réalisée en 2003-2006 dans le cadre d'une thèse de doctorat en anthropologie (Serra-Mallol 2007) sur les pratiques alimentaires et leurs représentations sociales dans l'archipel de la Société, auprès d'une quarantaine de ménages tahitiens ou ma'ohi résidant dans deux quartiers urbain et semi-urbain de Tahiti (Pamatai) et Moorea (Maatea).
} 
Tahiti des dix-neuvième et vingtième siècles, ou du «ma'a tahiti» à l'ère du C.E.P. Cela peut être également le cas de la consommation de certains mets jugés dégoûtants par d'autres cultures et notamment les cultures dominantes, et qui ont pu être interdits durant une longue période de colonisation, comme ce fut le cas avec la France en Polynésie française à propos de la consommation de viande de chien ou de tortue.

En Polynésie française, parmi le 'ina'i, l'accompagnement des végétaux, la «viande» est considérée comme supérieure au poisson pourtant jugé indispensable. La viande a une vertu de richesse et d'opulence que n'a pas le poisson. La chair animale terrestre est séparée en plusieurs catégories, la « viande » au sens où l'expriment les Tahitiens (la viande des mammifères, la chair rouge) et la viande de poulet. Il est intéressant de noter que cette séparation en deux classes suit l'ancienne dichotomie polynésienne carnée. La "viande » est largement préférée au poulet et constitue désormais, à la place du poisson, le complément carné ou 'ina 'i de base.

La viande de chien est souvent comparée aux autres «viandes rouges », comme le bœuf et le porc, et les femmes déclarent le plus souvent préférer le bœuf ou le porc au chien. La consommation de chien est observée surtout de la part des hommes: parmi les hommes interrogés, la majorité a déjà goûté la viande de chien. Elle nous a paru plus évoquée dans un quartier pourtant urbain (Pamatai), sans doute du fait de l'organisation $\mathrm{du}$ «fournisseur » principal qui en rend la disponibilité et donc l'occasion de consommer plus fréquentes : environ un cinquième des hommes du quartier urbain (Pamatai) en mangerait de façon occasionnelle mais régulière (une fois tous les mois à deux mois en moyenne), contre moins d'un dixième des hommes du quartier semi-rural (Maatea) étudié. Les personnes interrogées perçoivent cette consommation comme étant typique de l'archipel polynésien des Tuamotu, où elle était régulière il y a peu encore (Malcom et Massal 1955 : 24), et de la population chinoise de Tahiti dont sont issus selon nos interlocuteurs les meilleurs préparateurs de viande de chien. Cette remarque est à rapprocher du fait que Maatea constitue une communauté relativement peu mobile et fortement endogamique, alors que la totalité des personnes rencontrées à Pamatai n'est pas originaire de la commune mais des autres archipels et de celui des Tuamotu en particulier, et venue à Tahiti pour y trouver un emploi salarié.

En Polynésie française, la consommation contemporaine de chien n'est donc pas propre aux seuls habitants des Tuamotu parmi la population de souche. Igor de Garine notait déjà que dans l'archipel des Marquises les jeunes adultes procédaient à des festins de chien, qu'il analysait comme relevant de « la provocation et la dérision que ceux-ci affichent vis-à-vis de la société des adultes, y compris sur le plan alimentaire » (Garine 1981:44), et qui pourraient également se lire comme une volonté de s'affirmer en tant que Polynésiens face à l'acculturation perçue des adultes, liée à la monétarisation de l'économie à laquelle n'avaient pas encore accès ces jeunes gens.

Dans le quartier Pamatai à Faa'a, principale commune de l'agglomération tahitienne, des personnes passent de maison en maison pour proposer des plats préparés à base de viande de chien, s'adressant aux foyers dont ils connaissent l'appétence pour ce type de plat, et évitant soigneusement les maisons habitées par des Européens ou Popa'a. Le plat, constitué de viande de chien cuite dans son sang, une forme de civet agrémenté de gingembre et accompagné de riz, est vendu mille francs (8,30 euros environ) la portion de trois cents grammes environ, servie dans un contenant individuel en plastique transparent ou blanc, et recouvert d'une feuille de plastique alimentaire. A titre de comparaison, un plat complet populaire du type poulet fafa ou chao men 
est vendu environ huit cents francs cfp $\left(6,7\right.$ euros) dans les « roulottes ${ }^{9}$ » pour une portion unitaire d'environ trois cents à quatre cents grammes. Un autre moyen de comparaison est le transnational Big Mac, vendu 470 F cfp (3,9 euros) dans les deux restaurants tahitiens de la compagnie américaine, et le «menu Big Mac » composé d'une portion « moyenne » de frites et d'un verre «moyen » de soda, sans oublier les « sauces » (doses de mayonnaise et de ketchup distribuées gratuitement et dont les Polynésiens font grand cas), est au prix de huit cent vingt francs cfp (6,9 euros). Le prix relatif du plat à base de chien est donc plus élevé que des plats de type «populaire »: il ne s'agit donc pas d'un recours économique, ou d'un cas «d'urgence alimentaire », mais bien d'un acte délibéré de consommation d'un plat « de choix », festif et considéré comme «de luxe », consommé avec plaisir mais aussi discrétion quand l'occasion se présente. Quelques roulottes et snacks installés tout autour de l'île de Tahiti sont réputés pour servir du « ragoût de chien », les consommateurs habituels se passant le mot de bouche à oreille lorsque le plat réputé délicat est disponible.

Les chiens choisis pour être abattus sont en général des chiens errants, sans maître. Parmi les « préparateurs » de viande de chien rencontrés sur Tahiti, aucun ne nous a avoué procéder à de l'élevage ("il faut faire dans la discrétion »), même si les installations dans les cours jouxtant les habitations (niches fermées superposées à côté des ustensiles destinées à la cuisson) laissent penser le contraire, au moins en matière de «stockage » des animaux destinés à être mis à mort. Selon ces personnes, les chiens ne doivent pas avoir été vaccinés, et être considérés comme «propres » et «pas malades»: «il faut avoir l'œil pour voir si le chien est ma'i [malade]! ». Les chiens avec maîtres ne seraient donc pas «enlevés » ou «kidnappés » pour être consommés comme le prétend la rumeur à Tahiti, quoique cela ait pu arriver, mais prélevés parmi la population considérable de chiens errants qui pullulent sur l'île, et les bâtards préférés aux animaux de race plus prononcée qui ont plus de chances d'avoir été vaccinés. Les chiens destinés à être consommés sont de taille moyenne, le poil brun ou brun clair («jaune »), au poil court et au museau allongé.

Pour le préparer, le chien est mis à mort par strangulation avec une corde, et la nuque brisée («c'est plus rapide comme ça, et cela évite d'éveiller les soupçons du voisinage »), ou à coups de bâton sur le museau en le maintenant par la nuque. Le cadavre du chien est plongé quelques minutes dans l'eau bouillante, ou de l'eau bouillante versée sur lui, afin de détacher plus facilement les poils en grattant la peau avec un couteau, puis la bête est passée au chalumeau pour brûler et racler les poils restants, en la retournant plusieurs fois sur le sol ou en la suspendant à une corde. L'animal est alors soigneusement éventré pour récupérer le sang et les entrailles qui serviront à élaborer la sauce de cuisson, ainsi que les abats (cœur et foie), évitant que ces derniers ne soient pollués par le contenu des entrailles, l'urine ou la salive de l'animal. La tête est tranchée et jetée, ainsi que les pattes et la queue, et le sang récupéré dans un récipient. L'animal peut être alors «fumé », suspendu au-dessus d'un feu pour faire rougir la peau sans cuire pour autant la chair.

L'animal est ensuite coupé en petits morceaux et désossé, ou en fines lamelles «comme si c'était du veau à la broche », et les morceaux jetés dans une marmite "pour leur faire perdre leur $e a u$ ». Cette « eau » est récupérée, écumée et mise de côté. On ajoute aux morceaux de viande du

\footnotetext{
${ }^{9}$ Les roulottes sont des véhicules de type camionnettes, à l'arrière desquels est aménagée une cuisine servie par les côtés. Ambulants, leur activité est principalement nocturne. Près de trois cents roulottes sont installées en Polynésie française, dont 128 pour la seule île de Tahiti. Le client peut consommer un plat populaire et bon marché sur place, attablé devant un panneau amovible installé sur les côtés du véhicule et assis sur un tabouret amené par le « roulottier », ou dans quelques cas autour de tables en plastique, ou acheter des plats à emporter pour consommation à domicile
} 
gingembre frais, des feuilles de citron, de la peau de citron et parfois d'orange séchée, et un peu de sel, on mélange et on fait cuire entre un quart d'heure et une heure. L'eau écumée du chien est ajoutée alors, ainsi qu'un mélange d'anis étoilé broyé disponible dans les pharmacies chinoises de Tahiti. On remue et porte le tout à ébullition, puis le feu est de nouveau baissé et le sang du chien ajouté pour lier la sauce ainsi obtenue, dont l'assaisonnement est rectifié. Au bout du temps de cuisson de cette forme de civet, jusqu'à deux heures, la sauce est liée et la chair de l'animal a pris une couleur rouge sombre, comme le fait la chair de la tortue à la cuisson.

\section{2-2 Les conséquences réglementaires d'une consommation posée comme illégale}

Depuis $1959^{10}$, la viande de chien est interdite de consommation en Polynésie française car jugée viande « répugnante » (article 47), aliment «n'entrant pas normalement dans l'alimentation humaine tel que le chien etc...» (article 48), assertion répondant au comportement culturel métropolitain pourtant reprise par les législateurs polynésiens dans un souci de normalisation sociale et culturelle. Il est d'ailleurs intéressant que le seul exemple trouvé par le législateur soit justement celui du chien. D'après les textes, ces viandes doivent être saisies et détruites, et les personnes en cause font l'objet d'une amende au montant peu élevé.

Ce texte a été modifié par une nouvelle délibération en $1977^{11}$ qui omet de citer la viande de chien en tant que telle, sans non plus la lister dans « les animaux dont la chair est destinée à être livrée au public en vue de la consommation (...) et présentés à la vente » (art. 5), et qui doit être contrôlée par les services vétérinaires. La précision de la destination commerciale est importante, puisque l'article 7 précise « les animaux destinés en totalité à la consommation familiale peuvent être exemptés de cette inspection ». On peut ainsi déduire de ce texte que la viande de chien destinée à la consommation familiale est autorisée par défaut, la référence au caractère « répugnant» de la viande de chien étant désormais abandonnée, si les conditions de mise en oeuvre et notamment l'abattage sont respectées. Il faut attendre 2006 pour qu'un arrêté du Conseil des Ministres ${ }^{12}$ mette en application cette délibération pour préciser les conditions techniques de mise en œuvre des denrées animales pour leur vente. Mais outre les conditions générales d'installation, d'équipement et d'hygiène, il n'est fait aucune référence particulière à la viande de chien, sinon la seule référence aux «denrées qui ne sont pas reconnues propres à la consommation humaine ».

Malgré l'existence de la réglementation de 1959, les autorités publiques locales, constituées en grande majorité et jusqu'à peu de métropolitains expatriés, ont fait peu de cas de la consommation de chien, qu'ils considéraient comme une subsistance d'anciennes habitudes jugées «traditionnelles ». Mais la référence à la consommation de chien n'apparaît que rarement en tant que telle dans les documents officiels depuis cette date. Ainsi, l'enquête sur le budget des ménages polynésiens réalisée en 1966-67 (Brenez et Desnot 1998 :193) établit le montant des dépenses moyennes par mois et par unité de consommation pour la viande, en donnant le détail pour le mouton et l'agneau, le bœuf et le veau, le porc, les volailles, la charcuterie et les abats, mais en considérant un poste «autres viandes et non précisés » qui constitue plus de $30 \%$ du

\footnotetext{
${ }^{10}$ Délibération de l'Assemblée Territoriale ( ${ }^{\circ} 59-60$ du 16 octobre 1959 parue au J.O.P.F. le 30 novembre 1959) et arrêté $\mathrm{n}^{\circ} 1911$ du 06 novembre 1959 portant réglementation de l'abattage des viandes animales et l'inspection de denrées alimentaires d'origine animale.

${ }^{11}$ Délibération de l'Assemblée Territoriale n`77-116 du 14 octobre 1977 portant réglementation de l'inspection des denrées alimentaires d'origine animale, parue au J.O.P.F. le 15 décembre 1977.

${ }^{12}$ Arrêté $n^{\circ} 1115$ et 1116/CM du 6 octobre 2006 pris en application des articles 10 et 11 de la délibération ${ }^{\circ} 77-116$ AT modifiée par la délibération n $95-79$ AT du 23 mai 1995.
} 
total. Les autres enquêtes de consommation alimentaire ou de budget des ménages réalisées localement en 1977, 1986 et 2002 ne la font pas non plus apparaître.

L'une des rares exceptions notables est constituée par les rations alimentaires moyennes établies par les services du Commissariat à l'Energie Atomique pour le suivi des populations après les essais nucléaires aériens menés entre 1966 et 1974. Deux enquêtes alimentaires et nutritionnelles effectuées en 1965 et 1985 auprès d'adultes à Tureai (archipel des Tuamotu) et Tahiti font pourtant apparaître à Tureia et dans la zone rurale de Tahiti une consommation moyenne de 10 grammes par jour de viande de chien sur un total de trente grammes de viande (Ministère de la Défense 2006 : 268-269). On retrouve donc bien cette proportion de $30 \%$ de viande canine dans la consommation totale de viande, définie pudiquement comme «autre viande» dans les enquêtes statistiques officielles.

Devant ce qui est considéré comme une «survivance culturelle », les autorités publiques s'en tiennent au strict cadre de leurs missions. Ainsi, le Bureau de la Qualité Alimentaire et de l'Action Vétérinaire du Service du Développement Rural, en charge de l'inspection sanitaire des denrées animales, se limite à vérifier les conditions d'abattage des viandes vendues pour consommation humaine dans la restauration commerciale, la vente à emporter et à l'exportation, à partir des déclarations fournies par les commerçants eux-mêmes. Le Bureau ne possède pas de moyens de contrôle au-delà des documents de déclaration à l'importation et à l'exportation ou à l'abattoir, et les infractions constatées sont orientées vers la gendarmerie. Des contrôles sont également effectués pour vérifier que des espèces protégées ne font pas l'objet de détention et de commercialisation, en vertu d'un arrêté ${ }^{13}$ se basant sur le récent code de l'environnement. Les amendes existantes ne concernent d'ailleurs pas le type de viande saisi, mais les conditions d'abattage, l'animal devant être étourdi correctement et tué sans souffrance inutile. La seule référence à la viande canine est relevée dans la délibération de 1959, délibération jugée «non vivante » par les acteurs institutionnels, et dont les termes n'ont pas été repris depuis. De la même façon, le Centre d'Hygiène et de Salubrité Publique de la Direction locale de la Santé avoue son impuissance à intervenir en matière de consommation de chien, sauf sur commission du Procureur de la République, qui ne concerne que les violences faites aux animaux.

La consommation de chien est pourtant de notoriété publique, certaines roulottes sont réputées pour en vendre, mais avec discrétion, sans l'inscrire sur leur carte de menus, et les commerçants prennent la précaution de n'en proposer qu'aux personnes de connaissance. De même, les préparateurs de viande de chien prennent un soin particulier à ne pas attirer l'attention sur eux, afin de ne pas tomber sur le coup de la protection animale et du Code pénal. Le parallèle peut être fait ici avec la consommation d'ortolan en France, interdite et pourtant connue de tous, et pratiquée même avec délice par un des Présidents de la cinquième République française, tolérée donc quand elle ne sort pas du cadre privé.

Des associations de défense des animaux, créées par des métropolitains résidant en Polynésie française, luttent depuis peu contre la consommation et la commercialisation de la viande de chien en Polynésie, en s'appuyant sur la réglementation existante, et en centrant leur argumentation sur les tortures dont feraient l'objet les bêtes pour leur mise à mort mais en mélangeant à tort les phases de la préparation (en prétendant que le chien est ébouillanté avant que d'être tué), et en arguant des conditions sanitaires dans lesquelles l'abattage est réalisé,

\footnotetext{
${ }^{13}$ Arrêté $\mathrm{n}^{\circ}$ 0306/CM du 20 février 2008 modifiant les articles A 121-1 et suivants du code de l'environnement relatifs aux espèces, ajoutant une liste des espèces protégées, qui comptent parmi la faune : oiseaux et coquillages endémiques, raie manta, tortues olivâtre Lepidochelys Olivacea et caouanne Caretta Caretta, requins et mammifères marins à l'exception notable du requin Mako Isurus oxyrinchus.
} 
n'étant pas soumis à contrôle puisque interdit. Un des arguments également utilisé est l'impact que la consommation de chien, ou du moins son image, pourrait avoir sur les représentations des touristes étrangers, principale source de revenus propres du Territoire.

2-3 La perception de la viande de chien chez les Tahitiens aujourd'hui : goûts et dégoûts «L'alimentation, avec ses tabous et ses plats caractéristiques, est un moyen d'identifier les étrangers » (Goody 1984 : 239), de même que ses manières de table, ses goûts et ses dégoûts. Cette identification, vecteur de construction de l'identité ${ }^{14}$, est également lisible dans son contraire, la séparation, qui peut aller jusqu'à une forme de xénophobie culinaire qui peut se manifester par des moqueries (Garine 1996 : 21-22), comme celle à connotations sexuelles qui a cours à Tahiti à l'égard des Popa'a, des Européens, sur le taioro ${ }^{15}$ (Saura 2004: 109) dont l'insulte fait par antiphrase de la sauce marine au coco fermenté et à l'odeur prononcée, marqueur culturel tahitien, le synonyme péjoratif de «l'Autre», le français, le non circoncis (ou non supercisé). Le choix des aliments suit une ligne de partage entre nature et culture (Douglas 1979 : 145), l'expression du sentiment identitaire ma'ohi passe par la différenciation première en matière alimentaire et sexuelle.

Un des tests pour vérifier qu'un Popa'a est suffisamment intégré en Polynésie française est la consommation du fafaru $^{16}$, avec sa forte odeur de décomposition marine particulièrement désagréable aux narines occidentales. De même, manger du chien rapproche également de la «tradition » de la culture polynésienne, et notamment paumotu. Au-delà de l'aspect folklorique, ces consommations témoignent de la survivance d'un « goût » polynésien traditionnel.

Selon P. Rozin, les motivations du dégoût sont au nombre de trois : d'ordre sensoriel-affectif (goût, odeur, consistance...), d'ordre de l'anticipation (conséquences anticipées des résultats de l'ingestion) et de ce que nous connaissons des origines de l'aliment (Rozin 1995 : 98). Ainsi les Tahitiens avouent être dégoûtés par la viande de cheval ${ }^{17}$ et de lapin peut-être plus par ignorance car peu disponible dans les commerces, de chat sans doute pour leur proximité affective, et par le rat par anticipation de la perception sensorielle. Ils n'apprécient pas non plus les viandes à l'odeur trop forte à la cuisson comme le mouton, ni non plus les fromages au lait cru ou à l'odeur trop forte typiquement farani (français).

Les femmes rencontrées déclarent souvent avoir goûté de la viande de chien, mais à leur insu selon elles, et n'avouent pas de goût pour cette viande, contrairement aux hommes. De fait, le dégoût (faufau) réside plus dans le fait de manger un animal considéré par ailleurs comme domestique, que dans le goût et la saveur de la viande, jugés bons. Le dégoût vis-à-vis de la consommation de chien, qui provient en grande majorité des femmes, est lié à l'idée de la proximité affective de l'animal, considéré d'une manière générale comme un animal familier, voire un «ami ». Ainsi, comme l'a montré Jacqueline Milliet, «quelle que soit la société, on ne

\footnotetext{
${ }^{14}$ A propos de la consommation de durian, un fruit asiatique qui mûr dégage une odeur d'excrément, et de la décision culturelle qui fait de tout produit un produit consommable ou non, Matty Chiva (1993) écrit que «manger est une autre manière de faire partie d'un groupe, en adoptant ses règles, en faisant comme les autres pour être admis. L'enfant apprend d'abord, et surtout, par observation et en voulant faire comme les autres, comme les grands. De ce fait, l'aliment devient un élément majeur dans la construction de l'identité, personnelle, de groupe, culturelle ».

${ }^{15}$ Taioro ou taiero: amande de coco arrivée à maturité râpée et fermentée pendant un ou deux jours à l'aide d'ingrédients marins (têtes de chevrettes, coquillages) et mélangés parfois à un peu d'eau.

${ }^{16}$ Chair de poisson ou de crustacé mise à macérer pendant quelques jours dans une préparation liquide à base d'eau de mer.

${ }^{17}$ Une des remarques en forme de justification le plus souvent entendue de la part d'interlocuteurs tahitiens avouant leur goût pour la viande de chien était «vous les Farani, vous mangez bien du cheval ». Mangeurs de grenouille aux yeux des Anglais, les Français sont donc des « mangeurs de cheval » aux yeux des Tahitiens...
} 
mange pas son chien, on ne mange pas du chien, mais on mange un certain chien (...) Le goût et le dégoût se comprennent en fonction de l'attribution d'un territoire qui assigne aux animaux une distance - éloignement ou proximité - admises par tous les membres de la société » (Milliet 1995 : 88), comme l'illustre cette remarque relevée au cours du terrain d'enquête :

«Moi je ne mange pas du chien, je n'en mangeais pas avant. Il y a six mois je suis arrivée chez ma cousine (...) il était 11h30-12h00, ils étaient en train de manger, elle nous accueille et elle nous dit "ah, K. et T. venez goûter notre ma'a », on lui dit "c'est quoi ? », ben, elle a préparé du bon ma'a, on a tout mangé, et une fois qu'on a tout fini le plat, elle nous dit comme ça "vous savez ce que vous avez mangé " j'ai dit non, elle $m$ 'a dit «c'était du chien » Mais comme c'était bon, j'ai pas voulu recracher parce que c'était très bon. Il y a même des préparations, c'est bon. Mais depuis je ne mange plus le chien, parce que le chien est mon ami! (rire)»

La même réaction a été relevée à propos de la consommation de viande de tortue auprès des femmes rencontrées : "J'aime ça la tortue, mais ça fait mal quand je mange, c'est vrai, parce qu'une fois j'avais vu quelqu'un tuer une tortue, et j'avais vu son cour qui bougeait tout seul. J'en mange mais je n'aime pas trop ça. » ou encore «on aurait dit qu'elle pleurait quand on l'a tuée... ».

$\mathrm{Au}$ dégoût féminin sont souvent associées des idées de malaise physique : courbatures, rhumatismes dans les articulations..., qui reposent sur les vertus censées caractériser la viande de chien (viande forte, chaude...). Ces malaises, réels ou psychosomatiques, sont peut-être la trace des conséquences anciennes de la violation de tabous alimentaires.

La séparation chaud / froid est peut-être également liée à une séparation entre domaine masculin et domaine féminin. Ce sont les hommes en général qui s'activent à l'extérieur, au soleil, et autour du four enterré. De même, nous verrons que la consommation de viande de chien, viande jugée «chaude», qui «échauffe le corps», est formellement déconseillée aux femmes. L'attraction de la fraîcheur, et de l'intérieur des maisons par opposition à l'extérieur, semble donc être un des caractères polynésiens de la féminité, fraîcheur qui viendrait en contrepoint à la chaleur interne du corps humain qui produit et favorise la vie. Selon les personnes rencontrées, les préconisations de saison de consommation du chien sont la période «froide » de la saison sèche tropicale ou les jours de pluie. La nourriture de viande de chien n'est pas autorisée pour les enfants, considérée comme trop forte pour eux. Elle est déconseillée également aux personnes faibles ou malades, ou simplement fiévreuses. Des préceptes semblables à ceux de la médecine hippocratique paraissent donc s'appliquer en Polynésie, et mettent en parallèle les oppositions classiques chaud/froid et humide/sec (Anderson 1984) qui recoupent la séparation féminin/masculin dans le Pacifique (Barrau 1965), dans une classification des aliments et des mangeurs explicative des réactions de leurs corps. Outre ses vertus d'aliment «chauffant », la viande de chien est également réputée pour être fortifiante et aphrodisiaque, et se digérerait beaucoup plus facilement que le bœuf ou le porc, selon les hommes ma'ohi rencontrés.

Malgré le dégoût horrifié des Européens, manger du chien reste une façon de marquer sa différence, son appartenance à la culture polynésienne, qui est issue en partie de la fusion de deux grandes cultures cynophages : l'océanienne et l'asiatique. C'est aussi affirmer le côté festif du «manger ensemble», lorsqu'on prépare toutes les fins de semaine en commun des repas familiaux à base de chien sur les îlots coralliens en face de l'île de Huahine, ou lorsqu'on veut honorer non sans ironie des archéologues Popa'a (Occidentaux) en leur offrant quatre chiens cuits au ahima'a, le four enterré polynésien ${ }^{18}$. Consommer du chien, c'est affirmer son identité

\footnotetext{
${ }^{18}$ Interview de Bruno Saura, Les Nouvelles de Tahiti, 11 juillet 2003, p. 7.
} 
polynésienne, par un choix raisonné de signes caractéristiques, qui n'est parfois que la reprise d'un discours ethno-culturaliste que les acteurs tiennent eux-mêmes sur le sens de leurs pratiques (Babadzan 1999 : 29 ; Rigo 2003 : 205).

\section{Conclusion}

La «tradition » alimentaire tahitienne a connu plusieurs « grappes d'influence » depuis le contact avec les premiers Européens : christianisme (manières de table, absence de séparation à table, usage du pain), puis influences occidentales (bœuf, alcool, boite de conserves...) et asiatiques (sauces, préparations...), suivant plusieurs pôles culturels d'influence faisant de l'alimentation tahitienne contemporaine un système marqué par le polycentrisme et le syncrétisme. Elle s'est construite à la fois à partir du rejet des traditions anciennes et en continuité directe avec elles, en passant par une phase de «déconstruction culturelle» (Turgeon 2003: 200) préalable des populations par le colonisateur. La «tradition» tahitienne contemporaine n'est de fait que la résultante du système socio-économique précédant l'ère du salariat, l'intégration progressive par la société tahitienne d'éléments de modernité à la tradition ancienne pré-européenne, approche combinatoire et dynamique de la culture «qui confronte le passé et le présent, la tradition et la modernité et qui reste ouverte à toutes les influences et toutes les réélaborations d'où qu'elles viennent $\gg($ Kilani $1996: 32)$.

L'acculturation alimentaire est importante aujourd'hui à Tahiti, mais nous avons mis en évidence (Serra-Mallol 2007) la perpétuation de la dimension sociale et culturelle de l'alimentation et la persistance des structures culinaires traditionnelles. L'alimentation ne peut se réduire à son aspect strictement fonctionnel et nutritionnel, mais touche également à des aspects et des motivations qui ressortent de l'identité polynésienne, y compris dans la zone la plus urbanisée des Iles de la Société.

Les comportements autour de la nourriture et de la table subsistent longtemps malgré les changements radicaux intervenus dans la vie sociale. Ils sont donc susceptibles de jeter un éclairage assez particulier sur la culture d'une nation ou d'un groupe donné malgré l'impact de la colonisation, des systèmes de production industrielle alimentaire et du développement des techniques de conservation. Au-delà du processus d'incorporation individuelle, en mangeant l'homme s'incorpore dans un système culturel, où les modes de production et de consommation, la cuisine et les manières de table sont culturellement déterminés (Fischler 1990 : 66-70). L'alimentation est ainsi un élément central de la construction des identités : les particularismes alimentaires sont parmi les derniers « marqueurs » à disparaître, et l'alimentation saisie dans son contexte culturel peut être utilisée comme un puissant symbole d'identité, notamment en Polynésie (Pollock 1992 : 235), comme affirmation de sa cohésion interne et de son hétérogénéité par rapport aux cultures voisines, comme facteur de réalisation de soi par l'action, ou encore comme revendication identitaire passant par l'expression de types de consommation pouvant entrer en «résistance» avec ce qui est perçu comme venant de l'extérieur. Les Tahitiens ont préservé des plats et préparations qui leur sont spécifiques : poisson cru «à la tahitienne », porcelet au lait de coco, taioro, mitihue, po'e, fafaru, poulet fafa... Ces plats typiques sont de véritables marqueurs gustatifs, tous à base de lait de coco, qui signent la culture alimentaire tahitienne (Rozin 1995 : 105) et sont mobilisés dans les «démonstrations identitaires ». « Si l'aliment de base de la nourriture quotidienne paraît ne pas toujours résister comme marqueur identitaire, en revanche la nourriture festive est vécue tout à la fois comme un rappel de la 'tradition', et comme 'espace' et 'temps' où s'affirment des identités » (Garrigues-Cresswell et 
Martin 1998 : 14). La consommation de chien aujourd'hui à Tahiti ressort selon nous de ce phénomène identitaire.

\section{Bibliographie :}

ANDERSON Eugene N., 1984. Healing and Cooling. Foods Re-examined, Social Science Information, Sage, London

ANDERSON William, 1988. A Journal of a Voyage Made in His Majesty Sloop Resolution, in Beaglehole JC The Journals of Captain James Cook, pp. 721-986

BABADZAN Alain

- $\quad$ 1982. Naissance d'une tradition. Changement culturel et syncrétisme religieux aux Iles Australes (Polynésie française), ORSTOM, Travaux et Documents $n^{\circ} 154$, Paris

- 1999. L'invention des traditions et la nationalisme, Journal de la Société des Océanistes, Musée de l'Homme, Paris, n 109-2, pp. 13-35

BARRAU Jacques

- $\quad$ 1965. L'humide et le sec, an essay on ethnobiological adaptation to contrastive environments in the IndoPacific area, Journal of the Polynesian Society, vol. 74, p.329-346

- 1983. Les hommes et leurs aliments. Esquisse d'une histoire écologique et ethnologique de l'alimentation humaine, Messidor, Temps actuels, Paris

- 1991. L'homme et le végétal, in Poirier Jean, Histoire des Mœurs, Gallimard, Encyclopédie de la Pléiade, Tome I, Paris, pp. 1279-1306

BEAGLEHOLE J.C., 1988. The Journals of Captain James Cook, Hakluyt Society, Kraus Reprint, Extra Series $\mathrm{n}^{\circ}$ XXXVI, Millwood, New York, 8 volumes

BOUGAINVILLE Louis-Antoine de, 1982. Voyage autour du monde, Gallimard, Paris

BRENEZ J. et DESNOT J.P. Les budgets familiaux en Polynésie française 1967-68, Société d'Etudes pour le Développement Economique et Social, Tome II, Paris, décembre 1998

BUCK P.H., 1930. Samoan material culture, Bernice P. Bishop Museum Bulletin 75, Honolulu

CHIVA Matty, 1993. L'amateur de durian, in N'Diaye Catherine (dir.) La gourmandise. Délices d'un pêché. Ed. Autrement, Série Mutations/Mangeurs, novembre, Paris, pp. 90 à 96

COOK James, 1998. Relations de voyages autour du monde, La Découverte, Paris, 1978, rééd.

DOUGLAS Mary, 1979. Les structures du culinaires, Communications, Seuil, nº 31, Paris, pp. 145-170

DUMONT-D’URVILLE Jules Sébastien César, 1988. Voyage pittoresque autour du monde, Editions Haere Po, Tahiti

ELLEN Roy, 1999. Categories of Animality and Canine Abuse. Exploring contradictions in Nuaaulu Social Relationships with Dogs, Anthropos, 94, T. 1-3, Fribourg, pp. 57-68

ELLIS William, 1972. A la recherche de la Polynésie d'autrefois, Publications de la Société des Océanistes, $\mathrm{n}^{\circ} 25,2$ vol., Paris

FARB Peter et ARMELAGOS George, 1985. Anthropologie des coutumes alimentaires, Denoël, Paris 
FERDON Edwin N., 1981. Early Tahiti As The Explorers Saw It. 1767-1797, The University of Arizona Press, Tucson

FIRTH Raymond, 1957. We the Tikopia. A sociological study of kinship in primitive Polynesia, George Allen \& Unwin Ltd, London

FISCHLER Claude, 1990. L'Homnivore : le goût, la cuisine et le corps, Odile Jacob, Paris

FLANDRIN Jean-Louis, 1996. Alimentation et christianisme, Les Entretiens de Belley, Cahiers de l'OCHA, n 7 , http://lemangeur-ocha.com

GARINE Igor de

- 1981. L'ethnologie alimentaire des Marquises, in Ferembach Denise (dir.) Influence de la nutrition sur le phénotype et la physiologie de populations humaines, CNRS-DGRST, Paris, po. 34-45

- 1991. Les modes alimentaires : histoire de l'alimentation et des manières de table, in Poirier Jean, Histoire des Mœurs, Gallimard, Encyclopédie de la Pléiade, Tome I, Paris, pp. 1447-1627

- 1996. Introduction, in Bataille Benguigui MC. Et Couson F. (dir.), Cuisines, reflets des sociétés. Sépia, Musée de l'Homme, Paris, pp. 9-28

GARRIGUES-CRESWELL Martine et MARTIN Marie-Alexandrine, 1998. L'alimentation : entre mondialisation et expression identitaire, in Techniques et Cultures, Dynamique des pratiques alimentaires, Ed. de la Maison des Sciences de l’Homme, $\mathrm{n}^{\circ}$ 31-32, Paris, janvier-décembre, p. 1-16

GOODY Jack, 1984. Cooking, cuisine and class. A study in comparative sociology (Cuisines, cuisine et classes), Centre Georges Pompidou, Centre de Création Industrielle, coll. Alors, Paris

HANDY E.S.C. et HANDY Elizabeth Green, 1972. Native Planters in Old Hawaii : their Life, Lore and Environment, Bernie P. Bishop Museum, Bull. 233, Honolulu

HARRIS Marvin, 1987. The Sacred Cow and the Abominable Pig, Riddles of Food and Culture, Touchstone Book, New York

HENRY Teuira, 2000. Tahiti aux temps anciens, Publication de la Société des Océanistes, Musée de l'Homme, ${ }^{\circ} 1$, Paris

KAUFMANN Jean-Claude, 2004. L'invention de soi. Une théorie de l'identité, Armand Colin, Paris

KILANI Mondher, 1996. Introduction à l'anthropologie, Payot, Lausanne, $19923^{\text {ème éd. }}$

KIRCH Patrick V., 1991. Polynesian Agricultural Systems, in Cox Paul A. et Banack Sandra A. Islands, Plants, and Polynesians : An Introduction to Polynesian Ethnobotany, Dioscorides Press, Portland, pp. 113-133

LAHIRE Bernard, 2001. L'homme pluriel, les ressorts de l'action, Nathan, Paris

LAHLOU Saadi, 1998. Penser Manger : les représentations sociales de l'alimentation, PUF, Paris

LEACH Helen, 2003. Did East Polynesians have a concept of luxury foods ?, World Archaeology, Vol. 34 (3), pp. 442-457.

MALCOMS Sheila et MASSAL Emile, 1955. Etudes sur la nutrition et l'alimentation dans les établissements français de l'Océanie, Commission du Pacifique Sud, Document Technique n 85, Nouméa

MECHIN Colette, 1997. La symbolique de la viande, in Paillat Monique (dir.) Le Mangeur et l'Animal. Mutations de l'élevage et de la consommation. Ed. Autrement, Collection Mutations, juin, Paris, pp. 121-134 
MILLIET Jacqueline, 1995. Manger du chien ? C'est bon pour les sauvages, L’Homme, N 136, octobre-décembre, pp. 75-94

MINISTERE DE LA DEFENSE, 2006. La dimension radiologique des essais nucléaires français en Polynésie. A l'épreuve des faits, Rapport d'étude, Paris

MOLYNEUX Robert, 1988. Remarks in Port Royal Bay in King George the Third Island, in Beaglehole JC The Journals of Captain James Cook, pp. 551-554

MORRISON James, 1989. Journal de James Morrison, second-maître à bord de la Bounty, Publication de la Société des Océanistes $n^{\circ} 16,1996,3^{\text {ème }}$ éd.

OLIVER Douglas L. ,

- $\quad$ 1974. Ancient Tahitian Society, University Press of Hawaii, Honolulu, 3 vol.

- 2002. Polynesia in Early Historic Times, The Bess Press, Honolulu

PARKINSON Sydney, 1984. Journal of a Voyage in the South Seas in HMS Endeavour, Caliban Books, London

POLLOCK Nancy J., 1992. These roots remain : food habits in islands of the central and eastern Pacific since western contact, Institute for the Polynesian Studies, Laie-Hawai

PUJOL R. et CARBONE G., 1991. L'homme et l'animal, in Poirier Jean, Histoire des Mœurs, Gallimard, Encyclopédie de la Pléiade, Tome I, Paris, pp. 1307-1388

RAUST Philippe, 1986. Le chien, in Encyclopédie de la Polynésie française, Tome II, Christian Gleizal / Multipress, Papeete, pp. 98-101

REY-LESCURE Philippe, 1946. Le chien en Polynésie, B.S.E.O., Tome VII (N5), n 77, pp. 259-262

RIGO Bernard, 2003. Lieux-dits d'un malentendu culturel. Analyse anthropologique et philosophique du discours occidental sur l'altérité polynésienne, Au Vent des Iles, Papeete, $1^{\text {ère }}$ éd. 1997.

ROZIN Paul, 1995. Des goûts et des dégoûts, in Bessis Sophie (dir.) Mille et une bouches. Cuisines et identités culturelles. Autrement, Série Mutations/Mangeurs, N 154, mars, Paris, p. 96-105

\section{SAURA Bruno}

- 2003. Entre nature et culture. La mise en terre du placenta en Polynésie française. Haere Po, Papeete

- 2004. Dire l'autochtonie à Tahiti: Le terme ma'ohi : représentations, controverse et données linguistiques, Journal de la Société des Océanistes, Musée de l’Homme, Paris, n 119, 2/2, pp. 119-137

SCEMLA Jean-Jo, 1999. Le voyage en Polynésie. Anthologie des voyageurs occidentaux de Cook à Segalen, Robert Laffont, coll. Bouquins, Paris, 1994, rééd.

SERRA-MALLOL Christophe, 2007. Changement social et traditions alimentaires. Approche socio-anthropologique de l'alimentation à Tahiti (Iles de la Société, Polynésie française), Thèse de doctorat d'anthropologie, 2 vol., Université de la Polynésie française, Punaauia

TITCOMB Margaret, 1969 Dog and Man in the ancient Pacific, Bernie P. Bishop Museum, Special Publication 59, Honolulu, Hawaii

TURGEON Laurier, 2003. Patrimoines métissés. Contextes coloniaux et post-coloniaux, Ed. de la Maison des Sciences de l'Homme, les Presses de l'Université de Laval, Paris

WILSON James, 1968. A missionary voyage to the Southern Pacific Ocean 1796-1798, Frederick A. Praeger, New York, Washington and London 